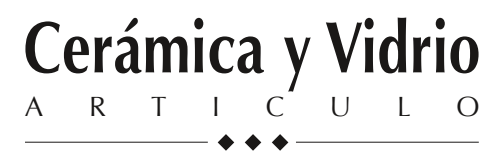

\title{
Olleros, alfareros y fabricantes de loza en Ribesalbes, entre 1742 y 1817
}

\author{
V. MARCELO-MARCO (*), M. COLLADO-LOZANO, I. NEBOT-DÍAZ \\ Escola Superior de Ceràmica de L'Alcora (ESCAL). L'Alcora (Castellón), España $(*)$ e-mail: vicky.marcelo@escal.es
}

\begin{abstract}
El desarrollo de la tradición alfarera en Ribesalbes, y el posterior establecimiento de las primeras fábricas de loza, a finales del siglo XVIII, viene determinado por los condicionantes naturales e históricos de la villa; así como por la influencia decisiva de la Real Manufactura del conde de Aranda, en la vecina población de L'Alcora.

En este trabajo, a partir de diversas fuentes documentales primarias, entre las que cabe destacar los libros sacramentales del Archivo Parroquial de Ribesalbes, y la Contribución general de 1817, se constata la presencia de olleros en la Baronía, a principios del siglo XVIII. Se determinan también los nombres de algunos alfareros, registrados en los albores del siglo XIX. Además se especifican los nombres de los trabajadores que aparecen vinculados a las fábricas de loza, existentes en 1817; estableciéndose la estructura profesional de la población de Ribesalbes, así como el porcentaje de vecinos contribuyentes vinculados a la cerámica.
\end{abstract}

Palabras clave: Fábricas de loza, olleros y alfareros, fabricantes, Joseph Ferrer, Ribesalbes siglos XVIII-XIX, Contribución general de 1817.

\section{Pottery and faience makers in Ribesalbes between 1742 and 1817.}

The development of the pottery tradition in Ribesalbes, and the later establishment of the first factories of tin-glazed earthenware, at the end of century XVIII, comes determined by the natural and historical conditioners from the villa; as well as by the decisive influence of the Real Manufacture of the count of Aranda, in the neighboring population of L'Alcora.

In this work, from diverse primary documentary sources, between which it is possible to emphasize sacramental books of the Parochial File of Ribesalbes and Contribución General de 1817, the presence of "olleros" in the Barony is stated, at the beginning of the XVIII century. The names of some potters, registered in the dawn of century XIX are also determined. In addition the names of the workers who appeared tied to the earthenware factories, existing are specified in 1817; settling down the professional structure of the population of Ribesalbes, as well as the percentage of neighboring tie contributors to the ceramics.

Keywords: Faience Factories, Pottery makers, Joseph Ferrer, Ribesalbes XVIII and XIX centuries,1817 General Contribution.

\section{INTRODUCCIÓN}

La población castellonense de Ribesalbes, ha sido tradicionalmente un centro productor de loza y de cerámica de aplicación arquitectónica. Configurando, históricamente, junto a sus vecinas, Onda y L'Alcora, el eje principal de la cerámica en la provincia de Castellón, desde el siglo XVIII.

Sin embargo, la ausencia de excavaciones arqueológicas en la zona, y la carencia de profundas investigaciones, que con fuentes documentadas analicen su cerámica desde un punto de vista histórico y artístico, evidencia la necesidad de abordar estudios especializados sobre el origen y desarrollo de la cerámica en la Baronía de Ribesalbes.

\section{OBJETIVOS}

Las referencias históricas documentadas sobre la loza en Ribesalbes son breves y sucintas reseñas (1); siendo las últimas y más recientes investigaciones las que han iniciado el estudio y vaciado de fuentes documentales primarias, para determinar las fábricas que coexistieron con la manufactura creada por el alcorino Joseph Ferrer, en 1780, o que se generaron a partir de ésta a principios del siglo XIX.

Por lo que este análisis pretende, por un lado, partiendo de las fábricas de loza cuya existencia se ha constatado entre 1780 y 1817, identificar a sus trabajadores. Por otra parte, verificar documentalmente la tradición alfarera de la Baronía con anterioridad a la creación de la fábrica de Joseph Ferrer.

\section{METODOLOGÍA}

El presente trabajo se fundamenta en el vaciado de los libros sacramentales del Archivo Parroquial de Ribesalbes y en el pormenorizado examen de la Contribución general de Ribesalbes de 1817, localizada en el Archivo Municipal de Ribesalbes.

Estas fuentes primarias consultadas resultan de vital importancia; pues al abordar el estudio de la cerámica en Ribesalbes resalta, como uno de los principales obstáculos, 
la carencia de fuentes documentales directas anteriores a la Guerra Civil española.

De hecho, la Contribución general de 1817 es el documento más antiguo de los conservados en el Archivo Municipal de Ribesalbes. Documento que permaneció desaparecido durante muchos años y que fue hallado, casualmente, durante la rehabilitación de la Casa de la Baronía, en 1992.

Respecto a los fondos del Archivo Parroquial de Ribesalbes hay que señalar que los libros sacramentales que se conservan, y que han sido consultados, recopilan los matrimonios, bautizos y defunciones de la Baronía a partir de 1741.

De ese modo, la documentación existente en ambos archivos acota cronológicamente los objetivos de la presente investigación.

Por otra parte hay que señalar que en la trascripción de los nombres, vocablos y datos extraídos de las fuentes documentales examinadas se han respetado en todo momento las grafías originales, así como las diversas variaciones que figuran y se recogen en dichos manuscritos.

\section{EL ORIGEN Y DESARROLLO DE LA TRADICIÓN CERÁMICA DE RIBESALBES}

Para el devenir cerámico de Ribesalbes resultan determinantes los condicionantes naturales e históricos que la convertirán en un núcleo, esencial y tradicionalmente, productor de loza y de cerámica de aplicación arquitectónica.

\subsection{Los condicionantes geográficos}

Etimológicamente, Ribesalbes procede de la contracción de dos palabras valencianas Ribes-albes que lo describen como "el de las blancas riberas". En clara alusión a sus características y blanquecinas tierras que conformarán la materia prima básica de su loza. De hecho, esta circunstancia ha sido reseñada en diversas descripciones (2) cuyos autores destacan la relevancia de la villa por la abundancia y calidad de sus minas de arcilla.

Así pues, las excelentes y abundantes minas de arcillas, la proximidad del agua del río Mijares en cuyos márgenes se asienta la Baronía y la abundancia de leña para los hornos, proveniente del abundante y típico monte bajo mediterráneo, son factores fundamentales y necesarios que facilitarán el nacimiento de una importante industria cerámica.

\subsection{Los antecedentes históricos: la alfarería de origen árabe}

Junto a los condicionantes naturales y geográficos de Ribesalbes, otro elemento primordial y decisivo será la tradición alfarera de la Baronía.

En Ribesalbes, hasta la fecha, no se ha podido acreditar con restos arqueológicos esa tradición alfarera, que fue tan propia de toda la zona levantina, en épocas antiguas y medievales (3). Sin embargo, la existencia de dicha tradición alfarera es más que probable. Pues Ribesalbes fue una alquería morisca, supeditada a la jurisdicción de Onda, bajo la dominación musulmana de la península, siendo evidente el origen morisco de la población de Ribesalbes.

Y no se debe olvidar que una de las grandes aportaciones de la población musulmana al reino de Valencia fue la cerámica, debido, principalmente, a su innata aptitud para este arte y a las excelentes arcillas que encontraron en las tierras levantinas, tal y como destacaron Bellver y Cacho (4) al estudiar la influencia de la dominación de los árabes en la provincia de Castellón. Además, esta elaboración cerámica de los árabes asentados en tierras valencianas perduró con el paso del tiempo tras la reconquista cristiana de este territorio. Los Fueros de Jaime I el Conquistador, otorgaron protección explícita a las "obras de tierra y de vidrio" (5), favoreciendo su producción.

Así pues, aún a falta de restos arqueológicos, parece innegable que la tradición alfarera de Ribesalbes se remonta a la influencia morisca, tal y como Sarthou reconoció (6); aludiendo, expresamente, a los ceramistas árabes como antecedentes de las manufacturas de Ribesalbes.

\subsection{La influencia de la Real Manufactura del Conde de Aranda}

Junto a los condicionantes naturales y geográficos de Ribesalbes y a la existencia de una tradición de alfarería popular, posiblemente dedicada a ollas y cántaros de uso doméstico, resultará determinante para la proyección cerámica de Ribesalbes, la creación en 1727, en la vecina población de L'Alcora, de la Real Manufactura de Cerámica por el noble aragonés, Don Buenaventura Pedro de Alcántara y Abarca de Bolea, IX conde de Aranda.

La loza de esta Real Manufactura alcorina fue tan apreciada; los operarios y artistas especializados que en ella se formaron y consolidaron fueron tantos y las condiciones laborales de la manufactura tan férreas $y$, en ocasiones, complicadas debido a disputas y rivalidades profesionales que, no es de extrañar que algunos de estos trabajadores especializados se aventurasen a crear sus propias empresas, tal y como recogió Escrivá de Romaní (7).

Por ello, bajo la directa influencia de la fábrica del conde de Aranda y a consecuencia de esta huida de operarios especializados, surgieron, a finales del siglo XVIII, tanto en L'Alcora como en poblaciones cercanas (Ribesalbes y Onda), "les fabriquetes" (8). Es decir, pequeñas fábricas de loza creadas por antiguos empleados de la Real Manufactura que centraron su producción en imitar los estilos y las piezas del conde de Aranda (9).

Una de estas fábricas de loza, creadas a finales del siglo XVIII, fue la que estableció en Ribesalbes Joseph Ferrer, uno de los más aventajados artistas de la Manufactura alcorina. Hombre polifacético y gran figura creativa que se formó bajo el mecenazgo del propio conde de Aranda, en la Academia de Bellas Artes de San Carlos, en Valencia. Fue miembro de la Academia de Nobles Artes de San Carlos de Valencia, pintor y escultor galardonado, reconocido y experto ceramista, propietario de una fábrica de loza en Ribesalbes, e Intendente de la Real Manufactura alcorina.

\section{LAS PRIMERAS MANUFACTURAS DE LOZA EN RIBE- SALBES}

\subsection{La fábrica de Joseph Ferrer}

Dadas, por una parte, las excepcionales condiciones naturales y geográficas de Ribesalbes ya señaladas, que proporcionaban una excelente arcilla, una abundante agua y un inagotable combustible, y evidenciada también, por otra parte, una tradición alfarera de origen árabe en el lugar, parece lógico que Joseph Ferrer eligiera Ribesalbes a finales del siglo 
XVIII, para ubicar su propia fábrica de loza. La manufactura de Joseph Ferrer en la Baronía, se encuentra documentada a partir de 1780 (10).

Pero además de las indudables cualidades ya citadas, Ribesalbes incorporaba otra gran ventaja: no formaba parte del señorío del conde de Aranda, y por tanto, la fábrica allí establecida se hallaba fuera de la jurisdicción directa del Conde (11), circunstancia ésta que, evidentemente, y vista la trayectoria de los distintos obradores que coetáneamente se establecieron en L'Alcora, resultó muy importante para la pervivencia de la fábrica de Ferrer.

La primera noticia que se conoce referente a la ubicación de esta fábrica de loza en Ribesalbes la ofrece Cavanilles (12), quien la describe como semejante a la de L'Alcora. En ella, Joseph Ferrer ejerció de arquitecto, maquinista y director. Y con él colaboraron, durante un tiempo, varios afamados artistas de la Fábrica alcorina, tal y como apuntan Ainaud de Lasarte (13) y Feliu (14), artistas entre los que cabe destacar a los pintores Álvaro, y a José y Cristóbal Mascarós. La maestría, pericia y relevancia de estos colaboradores avalaría la gran calidad de las piezas ribesalbenses.

Por todo lo que se deduce que la envergadura de la fábrica de Ferrer en Ribesalbes, debió ser ciertamente notable, hasta el punto de convertirse en una directa, importante $y$, sin duda, molesta rival para la de los Aranda.

\subsection{Otras fábricas de loza en Ribesalbes}

Pero la de Joseph Ferrer no fue la única fábrica de loza activa en Ribesalbes a finales del siglo XVIII, según analizan y concluyen los más recientes estudios (15), en los que, a partir de la Contribución general de Ribesalbes de 1817 y tras la investigación y examen de los expedientes referenciados por Todolí (16) vinculados a propietarios de fábricas de loza de Ribesalbes y dirigidos a la Junta General de Comercio y Moneda de Madrid en las primeras décadas del siglo XIX (17), se determinan algunos de los titulares de las fábricas de loza de principios del siglo Y se logra también precisar al propietario de una fábrica en Ribesalbes, a finales del siglo XVIII, coetáneo a Joseph Ferrer.

De forma que el análisis de dichas fuentes documentales permite configurar una visión global de la existencia de las primeras fábricas cerámicas en Ribesalbes, a finales del siglo XVIII y principios del siglo XIX

Determinándose que, contemporánea a la fábrica de Joseph Ferrer debió ser la del también alcorino Vicente Grangel Mesquida, quien en 1820, a la edad de sesenta y nueve años, aseguraba haber sido propietario de una fábrica de loza durante veinte años en la Baronía de Ribesalbes.

A principios del siglo XIX, concretamente en 1815, queda constatada la existencia de tres fábricas de loza en Ribesalbes, que se establecieron con anterioridad a 1811, según los citados expedientes. Fábricas cuyos titulares, vecinos de la Baronía, son: Joaquín Garcés y Benagues, Francisco Arsó (Arzo) y Ramón Bordonan (Bordonau) (18).

Estableciéndose a partir de 1811 dos fábricas más de loza, que, en base a la información extraída de la Contribución general de Ribesalbes de 1817, son la fábrica de Antonio Vicent y Bonet y la fábrica de la que son copropietarios Francisco Albalat, Josef Vicen y Bonet y Francisco Albalat y Doménech.

Por otra parte, en la citada Contribución general de 1817, se registran en total cinco fábricas de loza fin, en Ribesalbes, cuyos titulares son: Francisco Arzo y Ros, Joaquín Garcés y Benagues,
Jayme Balaguer y Compañía, así como los ya citados: Antonio Vicent y Bonet y los copropietarios Francisco Albalat, Josef Vicen y Bonet y Francisco Albalat y Doménech.

Una de estas fábricas, concretamente la de Jayme Balaguer y Compañía, se apunta como continuadora de la que fundó Joseph Ferrer en 1780, hipótesis que avala, por un lado, la vinculación laboral existente entre él y Jayme Balaguer (19) y por otro, el hecho de que sea ésta, con diferencia, la fábrica de mayor envergadura, valorada en 20.025 RV (reales de vellón).

\section{OLLEROS Y ALFAREROS EN LOS LIBROS SACRAMEN- TALES DEL ARCHIVO PARROQUIAL DE RIBESALBES}

Si bien hasta la fecha la fábrica de Joseph Ferrer ha sido la primera y única fábrica de loza de la que existía una constancia documentada y manifiesta; han quedado constatadas en las últimas investigaciones, tal y como se ha reseñado, las otras fábricas que se desarrollaron en sincronía y diacronía con aquélla.

Asimismo, se presuponía en Ribesalbes, como ya se ha señalado, la existencia de una artesanía popular alfarera, centrada en la elaboración de cántaros y ollas de uso doméstico y heredera de la tradición alfarera de origen musulmán. Sin embargo, no se había localizado ninguna constancia ni expresa, ni empírica, que avalase esta hipótesis (20).

En este sentido, la consulta de los libros sacramentales del Archivo Parroquial de Ribesalbes ha permitido obtener esenciales y novedosas informaciones, si bien no hay que olvidar que los fondos del citado Archivo se remontan a 1741.

Tras el minucioso vaciado de estos libros parroquiales se ha podido evidenciar, en el Libro de entierros (1741-1794), la existencia de un ollero de oficio llamado Joseph Peixó, casado con Mónica Balaguer, que fallece el 11 de septiembre de 1742, y cuya hija Rosa Peixó Balaguer, según consta en el Libro de velados (1741-1794), contrae matrimonio, el 30 de diciembre de 1743, con el labrador Jayme Bordonau Bonet, siendo todos naturales y vecinos de Ribesalbes.

En la correspondiente partida de defunción del citado libro de entierros no hay constancia de la edad que tenía el ollero en el momento de su fallecimiento, pero dado que un año después de su sepelio contrae matrimonio su hija (a la que hay que presuponerle en ese momento, según el patrón habitual de la época, una edad mínima aproximada entre dieciséis y veinte años), resulta lógico deducir que la edad de su padre, Joseph Peixó, rondaría como mínimo los cuarenta años, de forma que, en consecuencia, los inicios de su actividad como ollero deben retrotraerse, al menos, al primer cuarto del siglo XVIII. Con lo cual, Joseph Peixó debió iniciarse en el oficio de ollero incluso antes de la fundación en L'Alcora, en 1727, de la Real Manufactura de Cerámica por el IX conde de Aranda, siendo el desarrollo de su trabajo y su quehacer diario paralelo en el tiempo al de esta Fábrica alcorina hasta 1742, fecha en la que consta su fallecimiento.

Del segundo Libro de difuntos, que recopila los entierros a partir de 1795,se ha extraído el nombre de tres alfareros: Pasqual Albalat, Antonio Benedito y Manuel Bonet, a través de los registros de defunción de sus respectivas hijas.

El 30 de julio de 1801 se da sepultura en Ribesalbes a una niña, de nombre Francisca, hija de Pasqual Albalat, alfarero, y de su consorte Vicenta Masó. 
TABLA I: OLLEROS Y ALFAREROS DE RIBESALBES

\begin{tabular}{|c|c|c|}
\hline NOMBRE & OFICIO & AÑO \\
\hline Joseph Peixó & Ollero & 1742 \\
\hline Pasqual Albalat & Alfarero & 1801 \\
\hline Antonio Benedito & Alfarero & 1802 \\
\hline Manuel Albalat & Alfarero & 1802 \\
\hline
\end{tabular}

Por otra parte, la párvula Maria Rosa Benedito Masó, hija del alfarero Antonio Benedito, de veinticuatro años de edad y de su mujer Rosa Masó, de diez y nueve años, falleció de calentura el 16 de mayo de 1802, procediéndose a su entierro el 17 de mayo.

Y Joaquina Bonet Albalat, de trece meses de edad, hija de Manuel Bonet, alfarero, de cuarenta años, y de su esposa Manuela Albalat, de treinta y dos años de edad, falleció el 29 de mayo de 1802 de dentición.

Por lo que, estos tres alfareros, naturales y vecinos de Ribesalbes, Pasqual Albalat, Antonio Benedito y Manuel Bonet, cuyo oficio aparece consignado en el obituario parroquial en 1801 y 1802, son contemporáneos a la fábrica de loza de Joseph Ferrer.

\section{LA CONTRIBUCIÓN GENERAL DE 1817}

La ya citada Contribución general de 1817 es, como se ha indicado, el documento más antiguo de los conservados en el Archivo Municipal de Ribesalbes. En él figura la riqueza territorial, industrial y comercial que los vecinos de ésta y otras villas poseían en la Baronía, si bien, en dicho manuscrito no se especifican las partidas, ni los lindes de las propiedades, por lo que resulta imposible determinar la ubicación de los bienes reseñados dentro del término de Ribesalbes.

En el apartado de Riqueza Industrial se registran las cinco fábricas de loza fina ya citadas, asociadas a los contribuyentes que son titulares de las mismas: Francisco Arzo y Ros, Joaquín Garcés y Benagues, Antonio Vicent y Bonet, Jayme Balaguer y Compañía y los copropietarios Francisco Albalat, Josef Vicen y Bonet y Francisco Albalat y Doménech.

Además, este manuscrito contiene la relación de todos los contribuyentes especificándose el valor del impuesto que, por su profesión, debía satisfacer cada uno de ellos.

\subsection{Los oficios de los contribuyentes}

Casi todos los vecinos que aparecen relacionados en el documento tienen asignado junto al nombre, en el margen izquierdo de la hoja, su correspondiente oficio, lo que nos ofrece una valiosa información para establecer la estructura profesional de la población de la Baronía en 1817.

Se relacionan en la lista ciento quince nombres de contribuyentes, de los que dos, Francisco Albalat y Josef Vicent y Bonet, que son propietarios, cada uno de una tercera parte de una fábrica de loza, aparecen tachados y sin contribución alguna estipulada. Otros dos vecinos más, Cristoval Lecha Povil y Vicente Ten, aunque no aparecen tachados, tampoco tienen fijada ninguna contribución.

De estos cuatro vecinos mencionados, los dos primeros son
TABLA II: OFICIOS DE LOS CONTRIBUYENTES DE RIBESALBES EN 1817

\begin{tabular}{|c|c|}
\hline OFICIO & $\mathbf{N}^{\mathbf{0}}$ VECINOS \\
\hline Albañil & 2 \\
\hline Arrendada & 4 \\
\hline Carpintero & 1 \\
\hline Fabricante & 31 \\
\hline Herrero & 1 \\
\hline Jornalero & 4 \\
\hline Labrador & 64 \\
\hline Molinero & 1 \\
\hline Peinador de Cáñamo & 1 \\
\hline Sastre & 1 \\
\hline Sin especificar & 1 \\
\hline
\end{tabular}

fabricantes mientras que Vicente Ten figura como labrador y Cristoval Lecha Povil no tiene especificado oficio.

Para los ciento once vecinos restantes que figuran como contribuyentes, los oficios consignados son los de labrador, jornalero, sastre, herrero, albañil, carpintero, peinador de cáñamo, arrendada, molinero y fabricante (Tabla II).

El oficio de "arrendada" está vinculado, en los cuatro casos, a cuatro mujeres viudas (Maria Ten, Maria Arzo, Rosa Arzo y Josefa Benedito). Los oficios de albañil, carpintero, herrero, molinero y peinador de cáñamo son puntuales y los desempeña, normalmente, una sola persona (Tabla III), mientras que los oficios mayoritarios son el de labrador y el de fabricante.

Queda otro oficio, el de jornalero, que tienen estipulado cuatro vecinos (Cristoval Tena, Fran ${ }^{\text {co }}$ Vicent y Bonet, Jayme Moliner, y Josef Salvador y Edo), cuya contribución viene asignada literalmente "por su jornal", de lo que se deduce que son asalariados.

Dado que el término jornal, entre otras acepciones, es una medida de tierra, el término jornalero tiene la especificidad de trabajador agrícola. Por tanto, un jornalero debe entenderse como un asalariado del campo.

Por otra parte, Cristoval Bordonau no tiene adscrita actividad alguna en el margen izquierdo de su nombre, con lo

TABLA III: OFICIOS MINORITARIOS DE LOS CONTRIBUYENTES DE RIBESALBES EN 1817

\begin{tabular}{|c|c|}
\hline OFICIO & VECINO \\
\hline Albañil & $\begin{array}{c}\text { Pasqual Benedito y Arzo } \\
\text { Ramon Benedito }\end{array}$ \\
\hline Arrendada & $\begin{array}{c}\text { María Ten, Vda. } \\
\text { María Arzo, Vda. } \\
\text { Rosa Arzo, Vda } \\
\text { Josefa Benedito, Vda }\end{array}$ \\
\hline Carpintero & Joaq Benedito \\
\hline Herrero & Franco Tarasona $^{\text {co }}$ \\
\hline Jornalero & $\begin{array}{c}\text { Cristoval Tena } \\
\text { Franco Vicent yonet } \\
\text { Jayme Moliner } \\
\text { Josef Salvador y Edo } \\
\text { Crist. Santolaria }\end{array}$ \\
\hline Molinero & Josef Sansano \\
\hline Peinador de Cáñamo & Franco Albalat \\
\hline Sastre & \\
\hline
\end{tabular}


cual queda sin determinarse su oficio. Pese a que el concepto especificado para su contribución es por "Industria personal y una cavallería mayor".

De los dos oficios restantes, labrador y fabricante, el primero es el mayoritario con sesenta y cuatro vecinos que tienen asignada esta actividad, frente a los treinta y uno que figuran como fabricantes. Es decir, se registra el doble de contribuyentes labradores que de fabricantes.

Hay que puntualizar que uno de los vecinos (Cristoval Pexo), que tiene anotado en el margen izquierdo el oficio de labrador, tiene después detallado, al igual que todos los otros contribuyentes, el concepto de su tributo. Pero en este caso, curiosamente, no corresponde con el estipulado a priori, pues en el texto se determina "Por su oficio de fabricante y una caballería mayor", con lo cual, en principio, parece existir una contradicción al referenciarse ambos oficios para el mismo contribuyente.

Podría tratarse de un error, evidentemente, aunque también cabe la posibilidad de que compaginase ambas actividades, pues la contribución que paga, $165 \mathrm{RV}$, es mayor que la que abona la gran mayoría de labradores que también poseen una caballería mayor y que asciende, en estos casos, a 120 RV. Por tanto, estas cifras nos inducen a creer posible la hipótesis de que Cristoval Pexo, compatibilizase los dos oficios: el de labrador y el de fabricante.

\subsection{Los fabricantes}

Respecto al oficio de fabricante, en primer lugar se debe aclarar y determinar el alcance de su significado.

Hoy en día se define como fabricante a la persona que, por su cuenta, posee una fábrica. Evidentemente éste no es el significado que se debe sobrentender especificado para dicho término en la Contribución general de 1817. Pues si así fuese, encontraríamos treinta y una personas propietarias de fábricas en Ribesalbes, cosa que resulta, a todas luces, imposible.

Según figura en el texto del citado documento, las palabras que explican los conceptos de las contribuciones de los vecinos y que, en consecuencia, justifican la asignación de este oficio son: "Fabricante por la utilidad de su jornal", "Fabricante por la industria del jornal", "Fabricante por la industria personal de su oficio", "Por la industria del jornal de la fabrica [sic]", lo que, evidentemente, determina el significado que se le concede al término fabricante en ese contexto y que es el de trabajador de una fábrica.

De los treinta y un fabricantes que se relacionan (Tabla IV), tan sólo uno, Antonio Pastor, queda expresamente detallado como fabricante de loza, si bien, parece obvio que todos ellos estarían vinculados directamente a las fábricas de loza.

Contrastando esta relación de fabricantes (Tabla IV) con la de los titulares de fábricas de loza de Ribesalbes en 1817 (Tabla $\mathrm{V})$, se observa la coincidencia de dos nombres Jayme Balaguer y Joaquín Garses, mayor (o Joaquín Garces, ya que con ambas grafías aparece), de forma que ambos son empresarios y figuran también en la Contribución, con el oficio de fabricante.

Por el contrario, los propietarios Francisco Arzo y Ros, Antonio Vicent y Bonet y Francisco Albalat y Doménech, no figuran como fabricantes sino como labradores, por lo que de los sesenta y cuatro vecinos que constan como labradores, al menos tres de ellos están vinculados directamente a las fábricas de loza. Esto avala la hipótesis, ya expuesta, de que algunos de los contribuyentes compaginarían ambos oficios, es decir, conciliarían su actividad como labrador con la de fabricante,
TABLA IV: FABRICANTES DE LOZA EN 1817

\begin{tabular}{|c|c|}
\hline$N^{o}$ & FABRICANTE \\
\hline 1 & Jayme Oset y Bonet \\
\hline 2 & Jayme Balaguer \\
\hline 3 & Ramon Tarasona \\
\hline 4 & Josef Vicent y Arzo \\
\hline 5 & Fran $^{\mathrm{co}}$ Santolaria \\
\hline 6 & Cristoval Mallen Povill \\
\hline 7 & Salvador Arzo \\
\hline 8 & Luis Albalat \\
\hline 9 & Pasqual Albalat \\
\hline 10 & Salvador Oset \\
\hline 11 & Ramon Bordonau \\
\hline 12 & Josef Fornech \\
\hline 13 & Josef Aguilar \\
\hline 14 & Manuel Garces \\
\hline 15 & Joaquin Garces mayor \\
\hline 16 & Luis Aguilar \\
\hline 17 & Cristoval Oset y Bonet \\
\hline 18 & Eristoval Tarasena Manuel Aguilar md \\
\hline 19 & Antonio Benedito \\
\hline 20 & Josef Benedito \\
\hline 21 & Fran $^{\text {co }}$ Tena \\
\hline 22 & Vicente Mallen \\
\hline 23 & Josef Albalat y Mallen \\
\hline 24 & Josef Bonet y Ros \\
\hline 25 & Manuel Balaguer \\
\hline 26 & Cristoval Pastor y Santolaria \\
\hline 27 & Pasqual Benedito y Bordonau \\
\hline 28 & Manuel Bonet \\
\hline 29 & Josef Albalat y Arzo \\
\hline 30 & Miguel Albalat \\
\hline 31 & Antonio Pastor \\
\hline
\end{tabular}

bien como propietario propiamente dicho de una manufactura, o bien como asalariado de la fábrica de loza, siendo de suponer que esta circunstancia concurriría también en algún otro vecino.

Por su parte, Francisco Albalat, y Josef Vicent y Bonet, que son propietarios cada uno de una tercera parte de una fábrica de loza, como ya se ha explicado anteriormente, aparecen tachados y sin establecerles contribución alguna.

Así, considerando que los treinta y un vecinos que figuran como fabricantes están vinculados a las cinco fábricas existentes en Ribesalbes en 1817, sobre el total de ciento once contribuyentes relacionados, se obtiene un porcentaje de $27^{\prime} 9 \%$ de los vecinos vinculados al sector de la loza en la Baronía, frente a un $57^{\prime} 6 \%$ de labradores.

TABLA V: TITULARES DE FÁBRICAS DE LOZA EN 1817

\begin{tabular}{|c|c|}
\hline$N^{o}$ & PROPIETARIOS FÁBRICAS DE LOZA \\
\hline 1 & Jayme Balaguer y Compañía \\
\hline 2 & Fran $^{\text {co }}$ Arzo y Ros \\
\hline 3 & Joaquin Garses mayor \\
\hline 4 & Antonio Vicent y Bonet \\
\hline 5 & $\begin{array}{c}\text { Fran }^{\text {co }} \text { Albalat } \\
\text { Josef Vicen y Bonet } \\
\text { Franco }^{\circ 0} \text { Albalat y Doménech }\end{array}$ \\
\hline
\end{tabular}


Ahora bien, si a esos treinta y un vecinos se añaden los tres empresarios que figuran como labradores, el labrador que tiene también asignado el oficio de fabricante y consignamos también los dos empresarios que no están contabilizados como contribuyentes, la proporción se incrementa ligeramente. Seria de treinta y siete personas relacionadas con las fábricas de loza, de un total, en este caso, de ciento trece contribuyentes, con lo cual, el porcentaje de vecinos dedicados a la loza seria de 32' $7 \%$.

Por tanto, se puede concluir, por los datos extraídos y las cifras barajadas, que aproximadamente un mínimo del $30 \%$ de los contribuyentes mantenían, en 1817, una relación directa con las fábricas de loza en Ribesalbes. Unos como propietarios y otros como asalariados, a veces, combinando esa dedicación con la de labrador y en otras ocasiones como única actividad.

\subsubsection{LOS ALFAREROS DE 1801-1802 Y LOS FABRICANTES DE 1817}

Al contrastar la relación de los tres alfareros documentados en el citado libro de defunciones del Archivo Parroquial de Ribesalbes, entre 1801 y 1802 (Tabla I) con el listado de los treinta y un fabricantes (Tabla IV) que se extrae de la Contribución general de 1817, se observa que los tres nombres que figuran como alfareros en el obituario parroquial (Pasqual Albalat, Antonio Benedito y Manuel Bonet) constan como fabricantes, en 1817.

Dado que en 1801 y 1802, las edades de estos alfareros oscilan entre los veinticuatro y cuarenta años, es lógico pensar que los tres son los mismos que quince años después, en 1817, aparecen especificados como fabricantes.

\section{CONCLUSIONES}

Del exhaustivo vaciado de las fuentes consultadas y tras el consecuente análisis, estudio y confrontación de los datos obtenidos, se constata:

- La existencia en Ribesalbes de un ollero, Joseph Peixó, casado con Mónica Balaguer, que fallece el 11 de septiembre de 1742 cuya hija Rosa Peixó Balaguer contrae matrimonio el 30 de diciembre de 1743, con Jayme Bordonau Bonet, labrador. Ello avala la existencia de una artesanía alfarera en la villa, a principios del siglo XVIII.

- La presencia, entre 1801 y 1802, de tres alfareros en la Baronía: Pasqual Albalat, Antonio Benedito y Manuel Bonet, que son contemporáneos a la fábrica de loza de Joseph Ferrer.

- Que estos tres alfareros localizados entre 1801 y 1802 en la Baronía (Pasqual Albalat, Antonio Benedito y Manuel Bonet), figuran consignados como fabricantes en 1817.

- Los nombres de los treinta y un fabricantes vinculados a las cinco fábricas de loza existentes en Ribesalbes (Tabla IV), según la Contribución general de 1817.

- Que los dos oficios mayoritarios entre los vecinos de la Baronía, en base a la Contribución general de 1817, son el de labrador y fabricante, duplicando los labradores, al total de fabricantes registrados.

- Un mínimo del 30\% de los contribuyentes mantenía, en 1817, una relación directa con las fábricas de loza fina en Ribesalbes, unos como propietarios y otros como asalariados o fabricantes, siendo en algunos casos ésta su única actividad, mientras que en otros el oficio de fabricante se compaginaba con el de labrador.

\section{AGRADECIMIENTOS}

Para el desarrollo y elaboración de la presente investigación, han resultado esenciales las fuentes primarias localizadas y consultadas, tanto en el Archivo Municipal de Ribesalbes como en su Archivo Parroquial. Por lo que la autora y autores del artículo agradecen a la Corporación Municipal de Ribesalbes, presidida por su Alcalde, D. José Medina Lozano, y a D. Antonio Cepas Leal, cura párroco de esta población, todas las facilidades proporcionadas para acceder al estudio y análisis de los fondos documentales que custodian.

\section{NOTAS Y REFERENCIAS BIBLOGRÁFICAS}

1. P. Madoz. Diccionario geográfico-estadístico-histórico de España y sus posesiones de Ultramar, vol. XIII. Madrid: [s.n.], 1849 (Madrid: Imprenta del Diccionario geográfico-estadístico-histórico de Pascual Madoz). p. 468.

2. B. Mundina Milallave. Historia, geografía y estadística de la provincia de Castellón. Facs. de la ed. de: Castellón: Imprenta y Librería de Rovira Hermanos, 1873. Madrid: Confederación Española de Cajas de Ahorro, D.L.1988. p. 474.

3. V. Ortells Chabrera. "La industria cerámica a la Plana de Castelló. Tradició històrica i mundialització actual". Treballs de la Societat Catalana de Geografia. $2005, n^{\circ} 58$, p. 35-66.

4. M. Bellver; V. del Cacho. Influencia que ejerció la dominación de los árabes en la agricultura, industria y comercio de la provincia de Castellón de la Plana. Castellón: [s.n.], 1889. (Imp. F. Segarra). p. 181.

5. P. H. Taraçona. Institucions dels furs i privilegis del Regne de Valencia eo, summari e repertori de aquells. Valencia: en la eftampa de Pedro de Guete, 1580. p. 93

6. C. Sarthou Carreres. Geografía general del Reino de Valencia: provincia de Castellón. Facs. de la ed. de: Barcelona: Alberto Martín, 1913. Castellón: Caja de Ahorros y Monte de Piedad de Castellón, 1989. p. 224.

7. M. Escrivá de Romaní y de la Quintana. Historia de la cerámica de Alcora: estudio crítico de la fábrica, recetas originales de sus más afamados artífices, antiguos reglamentos de la misma. Facs. de la ed. de: Madrid: Aldus, 1945. Madrid: [s.n.], 1996. (Madrid: Imp. Fortanet). p. 182

8. Nombre con el que se denominó popularmente, y también en la documentación de la época, a las fábricas imitadoras.

9. Por lo que el conde de Aranda, en 1786, solicitó al rey Carlos III, que se cerrasen las fábricas competidoras, establecidas en L'Alcora; y que se marcasen los productos de las de Ribesalbes, Onda y Vall de Crist.

10. Museu de Ceràmica (L'Alcora). Museu de cerámica de L'Alcora: noves adquisicions 1998-2000 [Exposició]. L'Alcora: Ajuntament de L'Alcora, 2000. p. 18.

11. Ribesalbes era Baronía, y pertenecía a la familia Coll. Concretamente al barón, D. Jaime Coll; y posteriormente, a su hija, Da Inés Coll y Pastor, nacida en 1759 .

12. A. J. Cavanilles. Observaciones sobre la historia natural, geografía, agricultura, población y frutos del Reyno de Valencia, vol. I. Facs. de la ed. de: Madrid: Imprenta Real, 1795-1797. Valencia: [s.n.], 1975. (Valencia: Artes Gráficas Soler). p. 98.

13. J. Ainaud de Lasarte. Cerámica y vidrio. Madrid: Plus Ultra, 1952. p. 296.

14. J. Feliu Franch. "La cerámica arquitectónica de Onda en el siglo XIX". Director: Víctor Minguez Cornelles. [Tesis doctoral inédita]. Universitat Jaume I, Departament d'Història, Geografia i Art, Castelló de la Plana, 1998. p. 44

15. V. Marcelo Marco. Determinación y concreción de las fábricas de loza en Ribesalbes, durante la primera mitad del siglo XIX. [Texto inédito]. Trabajo de investigación para la obtención del DEA, Universidad Politécnica de Valencia, 2010

16. X. Todolí Pérez de León. La fábrica de cerámica del conde de Aranda en Alcora: Historia documentada, 1727-1858. Agost (Alicante): Asociación de Ceramología, 2002. p. 432-440 y p. 457-466.

17. El expediente con peticiones de tres fabricantes de Ribesalbes, dirigido a la Junta General de Comercio y Moneda de Madrid (1815-1816); y el expediente con la petición, dirigida a la Junta General de Comercio y Moneda de Madrid, en la que el duque de Hijar solicita la patente de la técnica del estampado de la loza, para su Fábrica de L'Alcora (1819-1820).

18. Los nombres se transcriben siempre con la grafía que se recoge en las fuentes consultadas. Existiendo, en muchos casos, pequeñas diferencias, dentro del mismo documento, que reflejamos.

19. V. Marcelo Marco; et al. "Determinación de las manufacturas de loza en Ribesalbes, entre 1780 y 1817". Arché. Publicación del Instituto Universitario de Restauración del Patrimonio de la Universidad Politécnica de Valencia. 2010, vol. 1, p. $199-210$

20. Desde finales del siglo $X X$, investigadores castellonenses, que parcialmente han abordado el estudio de la cerámica de Ribesalbes, como Francesc Esteve, Eugenio Díaz Manteca y Ferran Olucha Montins, entre otros, han planteado la necesidad de una aproximación documentada al patrimonio cerámico de Ribesalbes. Al igual que han hecho diversas instituciones y entidades culturales como el Museo de la Baronía del Ayuntamiento de Ribesalbes, y la Escola Superior de Ceràmica de L'Alcora.

Recibido: 30/11/2011

Aceptado: $24 / 08 / 2012$ 